\title{
THE SOLVABILITY OF OPERATOR EQUATIONS WITH ASYMPTOTIC QUASIBOUNDED NONLINEARITIES ${ }^{1}$
}

\author{
P. S. MILOJEVIĆ
}

\begin{abstract}
We study the solvability of operator equations involving quasibounded and asymptotically quasibounded nonlinear perturbations of linear Fredholm operators.
\end{abstract}

1. Let $X$ and $Y$ be Banach spaces, $L: X \rightarrow Y$ a linear Fredholm map of nonnegative index and $N: X \rightarrow Y$ a compact map. The operator equation of the form

$$
T x=A x+N x=f
$$

has been extensively studied by many authors in recent years. Under various growth conditions on $N$, the surjectivity of $T$ has been proven in a number of papers (see [4], [5], [7] and the references therein).

Alternatively, beginning with a paper of Landesman and Lazer [6], much work has been done on the solvability of equation (1) for a certain range of values of $P f$, where $P$ is the projection of $Y$ on the cokernel of $A$. Using the stable homotopy arguments, Nirenberg [9], [10], Berger [1], Mawhin [8], Podolak [11], Borisovich, Zvyagin and Sapronov [2] and others have studied equation (1). The alternative method has also been used to study equation (1) (with noncompact $N$ too) in a series of papers by Cesari and his coworkers, Fučik, Kučera and Nečas [5], and many others (cf. the survey paper by Cesari [3] and the monograph by Berger [1] for contributions of other authors). In all these papers (except in [2], [7], [11]) $N$ is assumed to have less than linear or linear growth.

In [2] and [11] the authors have studied equation (1) under the assumption that $N$ is asymptotically linear or asymptotically Lipschitz (i.e., $B$ in Definition 1 below is a Lipschitz map), respectively. In a series of papers Mawhin (cf. [7], [8]) has studied equation (1) with $f \in R(A)$ involving certain quasibounded maps $N$ using his coincidence degree.

In this paper we study the surjectivity of $T$ with $N$ either quasibounded or asymptotically quasibounded as defined below. Moreover, in case when the index of $A, i(A)$, is zero we provide a new growth condition on $\left.P N\right|_{\text {ker } A}$ that insures the solvability of equation (1) with these types of nonlinearities $N$. In the proofs of our main results we use a special case of the degree theory for

Received by the editors June 27, 1978.

AMS (MOS) subject classifications (1970). Primary 47H15.

${ }^{1}$ This work was partially supported by the NRC Grant A 4556.

(c) 1979 American Mathematical Society $0002-9939 / 79 / 0000-0420 / \$ 02.50$ 
compact perturbations of nonlinear $C^{1}$-Fredholm maps as developed in [2] or, equivalently, the stable homotopy arguments since for our map $T$ this degree can be defined in terms of elements of the stable homotopy group $\pi_{n+m}\left(S^{m}\right)$ (see [1], [2], [9]).

2. Set $X_{1}=\operatorname{ker} A$ and $Y_{2}=A(X)$. Since $A$ is Fredholm, $\operatorname{dim} X_{1}=n<\infty$ and $Y_{2}$ is closed we have the following direct sum decompositions: $X=X_{1} \oplus$ $X_{2}$ and $Y=Y_{1} \oplus Y_{2}$ with $\operatorname{dim} Y_{1}=m<\infty$ and $\operatorname{ind}(A)=n-m>0$. Define a new norm on $X$ by

$$
\|x\|_{1}=\max \left\{\left\|x_{1}\right\|,\left\|x_{2}\right\|\right\}
$$

where $x=x_{1}+x_{2}$ with $x_{i} \in X_{i}, i=1,2$. Let $P: Y \rightarrow Y_{1}$ be a linear continuous projection onto $Y_{1}, H$ be the inverse of the linear homeomorphism $\left.A\right|_{X_{2}}$ : $X_{2} \rightarrow Y_{2}$ and $\alpha=\|H\|$.

THEOREM 1. Suppose that for a given $f$ in $Y$ the following conditions hold:

(1) There exist constants $M_{f}>0$ and $N_{f}>0$ such that $P N\left(x_{1}+x_{2}\right)-t f_{1} \neq$ 0 for $\left\|x_{2}\right\|<r, r>N_{f},\left\|x_{1}\right\|>r M_{f}$ and $t \in[0,1]$;

(2) $M=H(I-P) N$ is quasibounded, i.e.,

$$
|M|=\limsup _{\|x\|_{1} \rightarrow \infty} \frac{\|M x\|}{\|x\|_{1}}<\infty
$$

and $|M| \max \left\{1, M_{f}\right\}<1$

(3) the stable homotopy class $\eta_{\rho}$ of $P N \mid S_{\rho}^{n-1}: S_{\rho}^{n-1} \rightarrow Y_{1} \backslash\{0\}, \rho>r M_{f}$, is nontrivial, where $S_{\rho}^{n-1} \subset X_{1}$ is a sphere of radius $\rho$.

Then equation (1) is solvable for this $f$.

Proof. Let $\varepsilon>0$ be small. By (2) there exists $R>N_{f}$ such that

$$
\|M x\|=\|H(I-P) N x\|<(|M|+\varepsilon)\|x\|_{1}
$$

for all $\|x\|_{1}>R$. Moreover, there exists an $r \geqslant R$ such that $A x+t(I-$ P) $N x-t f_{2} \neq 0$ for all $x=x_{1}+x_{2}$ with $\left\|x_{1}\right\|<r M_{f}$ and $\left\|x_{2}\right\|=r$ and $t \in[0,1]$. If not, then for each $r>R$ there exist $t \in[0,1]$ and $x$ with $\left\|x_{1}\right\|<r M_{f}$ and $\left\|x_{2}\right\|=r$ such that $A x_{2}+t(I-P) N x-t f_{2}=0$, and therefore

$$
\left\|x_{2}\right\| \leqslant\|H(I-P) N x\|+\alpha\left\|f_{2}\right\| \leqslant(|M|+\varepsilon)\|x\|_{1}+\alpha\left\|f_{2}\right\|,
$$

or

$$
1<\frac{1}{r}(|M|+\varepsilon)\|x\|_{1}+\frac{\alpha}{r}\left\|f_{2}\right\| \leqslant(|M|+\varepsilon) \max \left\{1, M_{f}\right\}+\frac{\alpha}{r}\left\|f_{2}\right\| .
$$

Passing to the limit as $r \rightarrow \infty$, we obtain $1<(|M|+\varepsilon) \max \left\{1, M_{f}\right\}$ which is in contradiction with condition (2) for $\varepsilon$ small enough. Hence, an $r$ with the above property exists.

Next, we define $\bar{D}=\left\{x=x_{1}+x_{2} \in X \mid\left\|x_{1}\right\|<r M_{f},\left\|x_{2}\right\|<r\right\}$ with $r$ chosen as above, and define the homotopy $H:[0,1] \times \bar{D} \rightarrow Y$ by

$$
H(t, x)=\left(A x+t(I-P) N x-t f_{2}, P N\left(x_{1}+t x_{2}\right)-t f_{1}\right) .
$$


We claim that $H(t, x) \neq 0$ for $t \in[0,1]$ and $x \in \partial D$. Indeed, if $x \in \partial D$ is such that $\left\|x_{2}\right\|<r$, then $\left\|x_{1}\right\|=r M_{f}$ and by (1), $P N\left(x_{1}+t x_{2}\right)-t f_{1} \neq 0$ for all $t \in[0,1]$. If $x \in \partial D$ is such that $\left\|x_{1}\right\|<r M_{f}$, then $\left\|x_{2}\right\|=r$ and $A x+t(I$ $-P) N x-t f_{2} \neq 0$ for all $t \in[0,1]$. Thus, by the homotopy theorem in [2],

$$
\operatorname{deg}(A+N-f, \bar{D}, 0)=\operatorname{deg}\left(H_{0}, \bar{D}, 0\right)=\eta_{r},
$$

which, by the solvability property of this degree, implies that $A x+N x=f$ for some $x \in D$.

To treat a larger class of nonlinear maps $N$, we need:

Definition 1. A map $N: X \rightarrow Y$ is said to be asymptotically quasibounded if there exists a nonzero continuous quasibounded map $B: X \rightarrow Y$, i.e.,

such that

$$
|B|=\lim _{\|x\| \rightarrow \infty} \frac{\|B x\|}{\|x\|}<\infty
$$

(A) $\lim _{R \rightarrow \infty} N(R x) / R=B(x)$ uniformly on bounded sets in $X$.

Such maps with $B$ Lipschitz have been studied by Podolak [11].

Theorem 1 admits the following extension:

Theorem 2. Suppose that $N$ satisfies condition (A) and that $B$ is continuous, satisfies conditions (1) and (3) of Theorem 1 for $f=0$ and that the following condition holds:

(2') $K=H(I-P) B$ is quasibounded, i.e.,

$$
|K|=\limsup _{\|x\| \rightarrow \infty} \frac{\|K x\|}{\|x\|_{1}}<\infty
$$

and $|K| \max \left\{1, M_{0}\right\}<1$.

Then equation (1) is solvable for each $f$ in $Y$.

Proof. Since for each $f$ in $Y, N_{f} x=N x-f$ satisfies condition (A) with the same $B$, it is sufficient to consider the case $f=0$. Define

$$
\bar{D}=\left\{x=x_{1}+x_{2} \in X \mid\left\|x_{1}\right\|<r M_{0},\left\|x_{2}\right\|<r\right\},
$$

where $r$ is chosen as in Theorem 1 using property $\left(2^{\prime}\right)$ of $K$. For $R>0$, define the $\operatorname{map} H_{R}: \bar{D} \rightarrow Y$ by

$$
H_{R}(x)=(1 / R)(A(R x)+(I-P) N(R x), P N(R x))
$$

and the homotopy $H:[0,1] \times \bar{D} \rightarrow Y$ by

$$
H(t, x)=\left(A x+t(I-P) B x, P B\left(x_{1}+t x_{2}\right)\right) \text {. }
$$

By our choice of $r$ we know that $H(t, x) \neq 0$ for $t \in[0,1]$ and $x \in \partial D$. Clearly, if $x \in X$ is a solution of equation (1), then $u=x / R \in D$ is a solution of $H_{R}(u)=0$ for $R$ sufficiently large, and conversely. Moreover, $\lim _{R \rightarrow \infty} H_{R}(x)=H(1, x)$ uniformly for $x \in D$ with $\|H(1, x)\|>\varepsilon>0$ for all $x \in \partial D$ since $H(1, \cdot)$ is a proper map. In view of this, it follows that for sufficiently large $R, H_{R}(x) \neq 0$ on $\partial D$ and

$$
F_{R}(t, x)=H(1, x)+t\left(H_{R}(x)-H(1, x)\right) \neq 0
$$


for $t \in[0,1]$ and $x \in \partial D$. The compactness of $N$ and condition (A) imply that $B$ is compact and consequently

$$
F_{R}(t, x)=A x+(1-t) B x+t N(R x) / R
$$

is an admissible homotopy on [0,1] $\times \bar{D}$ (cf. (4.2) in [2]). Hence,

$$
\operatorname{deg}\left(H_{R}, \bar{D}, 0\right)=\operatorname{deg}(H(1, \cdot), \bar{D}, 0)=\operatorname{deg}(H(0, \cdot), \bar{D}, 0)=\eta_{r}
$$

which implies that the equation $H_{R}(x)=0$ is solvable in $D$.

REMARK. When $N$ is asymptotically linear, i.e., $N(x)=B(x)+w(x), x \in$ $X$, for some continuous and linear map $B: X \rightarrow Y$ with $w(x) /\|x\| \rightarrow 0$ as $\|x\| \rightarrow \infty$, then $N$ is quasibounded with $|N|=\|B\|$. Hence, Theorem 1 extends Theorem 4.5 in [2], which is, on the other hand, an abstract extension of some results of Nirenberg [9] involving everywhere bounded nonlinearities $N$. Other extensions of Nirenberg's results to sublinear or quasibounded nonlinearities are given in [1], [4], [5], [7], [8] (cf. [1] for other references).

REMARK. If $B$ in condition (A) is Lipschitz, i.e., $\|B x-B y\|<k\|x-y\|$ for all $x, y \in X$ and some small $k>0$, then condition (1) in Theorem 2 can be replaced by the following easier to verify condition of Podolak [11]:

(1') $\left\|P N\left(a \cdot x_{0}\right)\right\|>b$ for some positive $b$ and all $a \in R^{n}$ with $\|a\|=1$, where $x_{0}=\left\{x_{01}, \ldots, x_{0 n}\right\}$ is a fixed basis for $\operatorname{ker} A$ of unit vectors and

$$
a \cdot x_{0}=a_{1} x_{01}+\cdots+a_{n} x_{0 n} .
$$

In this sense Theorem 2 extends Theorem 1 in [11].

Let us now look at a new condition on $\left.P N\right|_{X_{1}}$ which implies that $\operatorname{deg}\left(\left.P N\right|_{X_{1}}, B(0, r), 0\right) \neq 0$ with $B(0, r) \subset X_{1}$. Suppose that $X$ and $Y$ are such that there exist a map $J: X_{1} \rightarrow Y_{1}^{*}$ and a continuous and odd map $G$ : $X_{1} \rightarrow Y_{1}$ with $G x \neq 0$ for $x \neq 0$ and $(G x, J x)=\|G x\| \cdot\|J x\|$ for all $x \in X_{1}$. This is always so if $Y=X$ or $Y=X^{*}$. Indeed, if $Y_{1}=X_{1}$, as $G$ and $J$ we can take the identity and the normalized duality map, respectively; while, if $Y_{1}=X_{1}^{*}$ as $G$ and $J$ we can take the normalized duality map and the identity, respectively. The condition in question is:

(4) $\|P N x\|+(P N x, J x) /\|J x\|>0$ for $x \in \partial B(0, \rho), \rho>r M_{f}$.

Corollary 1. Let $A$ and $N$ satisfy conditions (1) and (2) of Theorem 1. Then, if condition (4) holds for all $\rho>r M_{f}$ and the index of $A$ is zero, equation (1) is solvable.

Proof. By Theorem 1 it suffices to show that $\operatorname{deg}(P N, B(0, \rho), 0) \neq 0$, where $P N$ is restricted to $\bar{B}(0, \rho)$. Define the homotopy $H:[0,1] \times \bar{B}(0, \rho) \rightarrow$ $Y_{1}$ by $H(t, x)=t P N x+(1-t) G x$. Then $H(t, x) \neq 0$ for $t \in[0,1]$ and $x \in \partial B$. If not, then $t P N x+(1-t) G x=0$ for some $t \in[0,1]$ and $x \in \partial B$. Since $t \neq 0,1$, we have

$$
\|P N x\|+\frac{(P N x, J x)}{\|J x\|}=\frac{1-t}{t}\|G x\|-\frac{1-t}{t} \frac{(G x, J x)}{\|J x\|}=0
$$


in contradiction with condition (4). By the oddness of $G$ we obtain:

$$
\operatorname{deg}(P N, B(0, \rho), 0)=\operatorname{deg}(G, B(0, \rho), 0) \neq 0 .
$$

Similarly, using Theorem 2, we obtain:

COROLlARY 2. Let $K$ be asymptotically quasibounded and $B$ satisfy conditions (1) and (2') of Theorem 2 with $f=0$. Then, if ind $A=0$ and $P B$ satisfies condition (4) for $f=0$, equation (1) is solvable for each $f$ in $Y$.

Under a somewhat stronger condition than (4), we have:

TheOReM 3. Let $X$ and $Y$ be Banach spaces with $\operatorname{dim} X=\operatorname{dim} Y<\infty$ and let $T: X \rightarrow Y$ be continuous and satisfy

(5) $\|T x\|+(T x, J x) /\|J x\| \rightarrow \infty$ as $\|x\| \rightarrow \infty$, where $J$ and $G$ are as above. Then $T(X)=Y$.

Proof. Let $f$ in $Y$ be fixed. By condition (5) there exists an $r_{f}>0$ such that

$$
\|T x-t f\|>0 \text { for }\|x\|=r_{f}, \quad t \in[0,1]
$$

and

$$
\|T x\|+\frac{(T x, J x)}{\|J x\|}>0 \text { for }\|x\|=r_{f} .
$$

The first inequality implies that

$$
\operatorname{deg}\left(T-f, B\left(0, r_{f}\right), 0\right)=\operatorname{deg}\left(T, B\left(0, r_{f}\right), 0\right),
$$

which is nonzero by the second inequality as shown in Corollary 1 . Hence, $T x=f$ is solvable.

REMARK. Along similar lines one can show that if $T: X \rightarrow X$ is continuous and compact (or condensing) and $I-T$ satisfies condition (5), then ( $I-$ $T)(X)=X$ (the proof will appear in a forthcoming paper by the author).

Condition (5) for $P N$ clearly holds if $P N$ is coercive on $X_{1}$, i.e.,

if $(P N x, J x) /\|J x\| \rightarrow \infty$ as $\|x\| \rightarrow \infty, x \in X_{1}$, or

if $(P N x, J x)>-c_{1}\|J x\|$ for all $x \in X_{1}$ and some $c_{1}>0$ and $\|P N x\| \rightarrow \infty$ as $\|x\| \rightarrow \infty, x \in X_{1}$, and, in particular,

if $\|P N x\|>c_{2}\|x\|^{k}$ for all $x \in X_{1}$ and some $c_{2}>0, k>0$.

The last condition holds if $N$ is $k$-homogeneous. Indeed, since $\|P N x\| \neq 0$ for $x \in \partial B(0, r) \subset X_{1}$,

$$
a=\min \{\|P N x\| \mid\|x\|=r\}>0
$$

and $\|P N x\|>\left(a / r^{k}\right)\|x\|^{k}$ for all $\|x\| \geqslant r$.

In view of the above discussion, we have the following special case of Theorem 2.1 in [8]:

TheOREM 4. Let $A: D(A) \subset X \rightarrow Y$ be a linear Fredholm map of index zero and $N: \bar{D} \subset X \rightarrow X$ a continuous compact map, where $D$ is open and bounded. Suppose that

(i) $A x \neq \lambda N x$ for $x \in D(A) \cap \partial D$ and $\lambda \in(0,1)$; 
(ii) $P N x \neq 0$ for each $x \in \operatorname{ker} A \cap \partial D$;

(iii) for some isomorphism $L: Y_{1} \rightarrow X_{1}$,

$$
\|L P N x\|+\frac{(L P N x, J x)}{\|J x\|}>0 \text { for } x \in \partial D \cap X_{1}
$$

with $J$ the normalized duality map from $X_{1}$ to $2^{X^{*}}$.

Then the equation $A x-\lambda N x=0$ has at least one solution in $D$ for each $\lambda \in[0,1]$.

Proof. It suffices to show (cf. [8]) that $\operatorname{deg}\left(\left.L P N\right|_{X_{1}}, D \cap X_{1}, 0\right) \neq 0$. But, this follows from condition (iii) as in Corollary 1 since $I$ is odd.

REMARK. The above results could be proven by using the homotopy

$$
H(\grave{t}, x)=\left(x_{2}+t H(I-P) N x-t f_{2}, P N\left(x_{1}+t x_{2}\right)-t f_{1}\right)
$$

instead. Hence, it is sufficient to require that the map $H(I-P) N: X \rightarrow X$ be compact or condensing. The same observation holds for Theorem 2 with $N$ replaced by $B$. Moreover, Theorem 2 of Podolak [11] can be shown to be valid for the nonlinearities considered in our Theorem 2 .

\section{REFERENCES}

1. M. S. Berger, Nonlinearity and functional analysis, Academic Press, New York, 1977.

2. Yu. G. Borisovich, V. G. Zvyagin and Yu. I. Sapronov, Nonlinear Fredholm mappings and Leray-Schauder theory, Uspehi. Mat. Nauk 32 (1977), 3-54. (Russian)

3. L. Cesari, Functional analysis, nonlinear differential equations and the alternative method, Lecture Notes in Pure and Appl. Math., Vol. 19, M. Dekker, New York, 1976, pp. 1-197.

4. S. Fucik, Nonlinear equations with noninvertible linear part, Czechoslovak Math. J. 24 (1974), 467-495.

5. S. Fučik, M. Kučera, and J. Nečas, Ranges of nonlinear asymptotically linear operators, J. Differential Equations 17 (1975), 375-394.

6. A. Landesman and C. Lazer, Nonlinear perturbations of linear elliptic boundary value problems at resonance, J. Math. Mech. 19 (1970), 609-623.

7. J. Mawhin, The solvability of some operator equations with a quasibounded nonlinearity in normed spaces, J. Math. Anal. Appl. 45 (1974), 455-467.

8. Topology and nonlinear boundary value problems, Dynamical Systems, Vol. 1, (Edited by L. Cesari, J. K. Hale and J. P. La Salle), Academic Press, New York, 1976, pp. 51-83.

9. L. Nirenberg, An application of generalized degree to a class of nonlinear problems, Troisième Colloq. d'Analyse Fonctionelle, (Liège, 1970), Centre Belge de Rech. Math., pp. 57-74.

10. __ Topics in nonlinear functional analysis, Courant Inst. of Math. Sciences, New York, 1974.

11. E. Podolak, On asymptotic nonlinearities, J. Differential Equations 26 (1977), 69-79.

Department of Mathematics, Université d’Ottawa, Ottawa, Ontario, Canada KiN 6N5

Current address: Departamento de Matemática, Universidade Federal de Minas Gerais, 30.000 Belo Horizonte, Brasil 